\title{
Admissibility Estimation of Pareto Distribution Under Entropy Loss Function Based on Progressive Type-II Censored Sample
}

\author{
Guobing Fan \\ Department of Basic Subjects, Hunan University of Finance and Economics, Changsha, China
}

\section{Email address:}

fanguobingmath@163.com

\section{To cite this article:}

Guobing Fan. Admissibility Estimation of Pareto Distribution Under Entropy Loss Function Based on Progressive Type-II Censored Sample. Pure and Applied Mathematics Journal. Vol. 5, No. 6, 2016, pp. 186-191. doi: 10.11648/j.pamj.20160506.13

Received: October 5, 2016; Accepted: October 14, 2016; Published: November 7, 2016

\begin{abstract}
The aim of this paper is to study the estimation of Pareto distribution on the basis of progressive type-II censored sample. First, the maximum likelihood estimator (MLE) is derived. Then the Bayes estimator of the unknown parameter of Pareto distribution is derived on the basis of Gamma prior distribution under entropy loss function. Further the empirical Bayes estimator also obtained by using maximum likelihood on the basis of Bayes estimator. Finally, the admissibility of a class of inverse linear estimators are discussed under suitable conditions.
\end{abstract}

Keywords: Admissibility, Bayes and Empirical Bayes Estimators, Progressive Type-II Censored Sample, Entropy Loss Function

\section{Introduction}

In practical life testing experiments, considering with the time limitation and/or other restrictions (such as cost, material resources, etc.), the experimenter may not always be in a position to observe the life times of all the products in a lifetime test $[1,2]$. Censored samples often arise in practice. Progressive Type II censored sampling test is one of the most important method of obtaining data in lifetime researches. The statistical inference studies for various distributions when sample belongs to progressive censoring have attracted many authors' attention. For example, Ng et al. [3] computed the expected Fisher information and the asymptotic variancecovariance matrix of the ML estimates based on progressively Type-II censored sample from Weibull distribution They also discussed the construction of progressively censored reliability sampling plans. Soliman et al. [4] investigated the point and interval estimations for the modified Weibull distribution based on progressively type-II censored sample. Yang [5] derived the maximum likelihood estimation of Weibull distribution under Type II progressive censoring with random removals, where the number of units removed at each failure time follows a binomial distribution. $\mathrm{Wu}[6]$ considered the estimation problem of the two-parameter bathtub-shaped lifetime distribution based a progressively type-II censored sample. Cho et al. [7] discussed the Bayes estimation of the entropy of a twoparameter Weibull distribution based on the generalized progressively censored sample. Bhattacharya et al.[8] proposed an optimum life-testing plans under Type-II progressive censoring scheme using variable neighborhood search algorithm. Laumen and Cramer [9] discussed the likelihood inference and statistical test procedure for the lifetime performance index in the presence of progressive censoring. Khorram and Farahani [10] considered the maximum likelihood estimation and bayes estimation of parameters of weighted exponential distribution based on progressively Type-II censored sample.

Many statistical inference problems have been discussed for various lifetime distributions, such as exponential distribution, Weibull distribution, etc. Wu and Chang [11] pointed out that Pareto distribution can be regarded as a suitable alternative distribution in modeling product's lifetime. Statistical inference about Pareto distribution receives great attention by authors in recent yeasrs. For example, Raqab et al. [12] derived the best linear unbiased 
predictors, maximum likelihood predictors and approximate maximum likelihood predictors of times to failure of units censored from Pareto distribution. Kulldorff and Vannman [13] obtained the best linear unbiased estimates based on the complete sample and the asymptotically best linear unbiased estimates based on a few selected order statistics. Fu et al. [14] discussed the Bayesian estimation of Pareto distributions under progressive Type-II censoring on the basis of several types of noninformative priors, i.e. Jeffreys prior, two reference priors and two general forms of second order probability matching prior. Saldaña-Zepeda et al. [15] proposed a goodness of fit test procedure for the Pareto distribution, when the observations drawn from Type II right censoring.

Assume that the repair time $X$ follows the Pareto distribution with the following probability density function (pdf) and cumulative distribution function (cdf) respectively:

$$
\begin{array}{cc}
f(x ; \theta)=\theta x^{-(\theta+1)}, & x>1, \theta>0 \\
F(x ; \theta)=1-x^{-\theta}, & x>1, \theta>0
\end{array}
$$

This paper will discuss the Bayes estimation of the parameter of Pareto distribution under entropy loss function on the basis of progressive type-II censored sample. The admissibility of estimators is an important topic [16-19]. Thus this paper will also study the admissibility and inadmissibility of a class of inverse linear estimators under suitable conditions. The remains of this paper are organized as follows. The MLE and Bayesian estimators of the parameter are obtained in Section 2. In Section 3, the admissibility and inadmissibility of estimators with inverse linear form are discussed. A conclusion is finally made in Section 4.

\section{Preliminary Knowledge}

The progressively Type II censoring scheme can be described as follows [20].

First, the experimenter places $\mathrm{n}$ units or individual on test.

(i) When the first failure is observed at $X_{1: m: n}$, then randomly select $r_{1}$ surviving unites and remove them.

(ii) When the i-th failure unit is observed at $X_{i: m: n}$, then randomly select $r_{i}$ surviving unites and remove them. $\mathrm{i}=2$, $3, \ldots, \mathrm{m}$.

(iii) This experiment terminates when the m-th failure unit is observed at $X_{m: m: n}$ and $r_{m}=n-m-r_{1}-\cdots-r_{m-1}$ of surviving units are all removed. Here the censoring numers $r_{1}, r_{2}, \cdots, r_{m-1}$ are all pre-fixed.

Remark 1. Note that if $r_{1}=r_{2}=\cdots=r_{m-1}=0, r_{m}=n-m$, This censored scheme reduces to conventional type II right censoring scheme. Also note that if $r_{1}=r_{2}=\cdots=r_{m-1}=r_{m}=0, m=n$, the progressively type II censoring scheme reduces to the complete sample case.

This paper we always assume that
$X=\left(X_{1: m: n}, X_{2: m: n}, \cdots, X_{m: m: n}\right)$ is a progressively Type-II censored sample from a life test on $\mathrm{n}$ items whose lifetimes follows Pareto distribution with pdf shown in (1), $x=\left(x_{1: m: n}, x_{2: m: n}, \cdots, x_{m: m: n}\right)$ is the corresponding observation of $X$ and $r_{1}, r_{2}, \cdots, r_{m}$ denote the corresponding numbers of units removed from the test.

\subsection{Maximum Likelihood Estimation}

The likelihood function of $\theta$ under given progressively type-II censored sample $x=\left(x_{1: m: n}, x_{2: m: n}, \cdots, x_{m: m: n}\right)$ is (Balakrishnan and Aggrwala [21]):

$$
L(\theta \mid x)=c \prod_{i=1}^{m} f\left(x_{i} ; \theta\right)\left[1-F\left(x_{i} ; \theta\right)\right]^{r_{i}}
$$

Here $x_{i} \equiv x_{i: m: n}$ is the observation of $X_{i: m: n}, i=1,2, \cdots, m$, $f(x ; \theta)$ and $F(x ; \theta)$ are given respectively by (1) and (2), and $c=n\left(n-r_{1}-1\right) \cdots\left(n-r_{1}-r_{2}-\cdots-r_{m-1}-m+1\right)$.

Substituting (1) and (2) into (3), the likelihood function is given by

$$
\begin{aligned}
L(\theta \mid x) & =c \prod_{i=1}^{m} \theta x_{i}^{-(\theta+1)} \cdot\left[x_{i}^{-\theta}\right]^{r_{i}} \\
& =c \theta^{m}\left(\prod_{i=1}^{m} x_{i}^{-1}\right) \cdot \exp \left[-\theta \sum_{i=1}^{m}\left(1+r_{i}\right) \ln x_{i}\right]
\end{aligned}
$$

Where $x_{i} \equiv x_{i: m: n}$ is the observation of $X_{i: m: n}, i=1,2, \cdots, m$ The natural logarithm of likelihood function is given by

$$
\ln L(\theta \mid x)=\ln c+m \ln \theta+\sum_{i=1}^{m} x_{i}^{-1}-\theta \sum_{i=1}^{m}\left(1+r_{i}\right) \ln x_{i}
$$

Then the MLE of $\theta$ can is the solution of the following equation

$$
\frac{d \ln L(\theta \mid x)}{d \theta}=\frac{m}{\theta}-\sum_{i=1}^{m}\left(1+r_{i}\right) T\left(x_{i}\right)=0
$$

Thus the MLE of $\theta$ can be easily solved as

$$
\hat{\theta}_{M L E}=\frac{m}{\sum_{i=1}^{m}\left(1+r_{i}\right) T\left(X_{i: m: n}\right)}
$$

\subsection{Bayes Estimation}

This subsection will discuss the Bayes estimation of the parameter $\theta$ of Pareto distribution (1) based on progressively Type-II censored sample under the following entropy loss function:

$$
L(\hat{\theta}, \theta)=\frac{\hat{\theta}}{\theta}-\ln \frac{\hat{\theta}}{\theta}-1
$$

Lemma 1. Let $X=\left(X_{1: m: n}, X_{2: m: n}, \cdots, X_{m: m: n}\right)$ be a progressively type-II censored sample from a life test on $n$ 
items whose lifetimes have Pareto distribution (1), and $r_{1}, r_{2}, \cdots, r_{m}$ denote the corresponding numbers of units removed from the test. Then under the entropy loss function (8), the unique Bayes estimator $\hat{\theta}_{B}$ of $\theta$ is (Wang et al. [22]):

$$
\hat{\theta}_{B}=\left[E\left(\theta^{-1} \mid X\right]^{-1}\right.
$$

Suppose the prior distribution of $\theta$ is Gamma distributions, $\Gamma(\alpha, \beta)$, with the following pdf

$$
\pi(\theta \mid \alpha, \beta)=\frac{\beta^{\alpha}}{\Gamma(\alpha)} \theta^{\alpha-1} e^{-\beta \theta}, \theta>0
$$

where $\alpha>0$ and $\beta>0$.

It is easy to proved that the posterior distribution of $\theta$ is also a Gamma distribution $\Gamma(m+\alpha, t+\beta)$, i.e. $\theta \mid X \sim \Gamma(m+\alpha, t+\beta)$,where $t=\sum_{i=1}^{m}\left(1+r_{i}\right) \ln x_{i}$ and from Lemma 1 , the unique Bayes estimator $\hat{\theta}_{B}$ can be obtained as

$$
\hat{\theta}_{B}=\left[E\left(\theta^{-1} \mid X\right)\right]^{-1}=\left(\frac{T+\beta}{m+\alpha-1}\right)^{-1}
$$

Where $T=\sum_{i=1}^{m}\left(1+r_{i}\right) \ln X_{i: m: n}$

The Bayes estimator $\hat{\theta}_{B}$ can be rewritten as

$$
\hat{\theta}_{B}=\left(\frac{1}{m+\alpha-1} T+\frac{\beta}{m+\alpha-1}\right)^{-1}
$$

which has of the form $(c T+d)^{-1}$

Remark 2 The Bayes estimator in Eq. (11) is seen to depend on the prior parameter $\beta$. Then When the parameter $\beta$ is unknown, we can use the empirical Bayes approach to estimate it. From (1) and (10), the marginal pdf of $X$ can be derived as follows

$$
\begin{aligned}
& m(x \mid \beta)=\int_{0}^{\infty} f(x \mid \theta) \pi(\theta \mid \beta) d \theta=\int_{0}^{\infty} L(x \mid \theta) \pi(\theta \mid \beta) d \theta \\
& =c \theta^{m}\left(\prod_{i=1}^{m} x_{i}^{-1}\right) \cdot \exp \left[-\theta \sum_{i=1}^{m}\left(1+r_{i}\right) \ln x_{i}\right] \frac{\beta^{\alpha}}{\Gamma(\alpha)} \theta^{\alpha-1} e^{-\beta \theta} d \theta \\
& =c \frac{\beta^{\alpha}}{\Gamma(\alpha)} \frac{\Gamma(m+\alpha)}{(\beta+t)^{m+\alpha}} \prod_{i=1}^{m} x_{i}^{-1}
\end{aligned}
$$

Then based on $m(x \mid \beta)$, we can get MLE of $\beta$ as

$$
\hat{\beta}=\frac{\alpha}{m} T
$$

Substituting $\hat{\beta}$ for $\beta$ in the Bayes estimator (12), the empirical Bayes estimator of $\beta$ can obtained

$$
\begin{aligned}
\hat{\theta}_{E B} & =\left(\frac{1}{m+\alpha-1} T+\frac{1}{m+\alpha-1} \frac{\alpha}{m} T\right)^{-1} \\
& =\left[\frac{m+\alpha}{m(m+\alpha-1)} T\right]^{-1}
\end{aligned}
$$

\section{Admissibility and Inadmissibility of $(c \boldsymbol{T}+\boldsymbol{d})^{-1}$}

In the following discussion, we always supposes that $X=\left(X_{1: m: n}, X_{2: m: n}, \cdots, X_{m: m: n}\right)$ is a progressively Type-II censored sample from a life test on $n$ items whose lifetimes have Pareto distribution (1), and $r_{1}, r_{2}, \cdots, r_{m}$ denote the corresponding numbers of units removed from the test.

Note that the MLE, Bayes estimator and empirical estimators obtained in Section 2 are all the special cases of a class of inverse linear estimators of the form $(c T+d)^{-1}$. In the rest of this section, the admissibility of these estimators will be discussed on the basis of their risks under the entropy loss function (8). Let $c^{*}=\frac{1}{m+1}, T=\sum_{i=1}^{m}\left(1+r_{i}\right) \ln X_{i: m: n}$.

For later use, we need the following result shown in Lemma 2.

Lemma 2. The statistics $T=\sum_{i=1}^{m}\left(1+r_{i}\right) \ln X_{i: m: n}$ has Gamma distribution $\Gamma(m, \theta)$.

Proof. Let $Y_{i}=\theta \ln X_{i: m: n}, i=1, \cdots, m$, then we can easily proved that $Y_{1}<\cdots<Y_{m}$ is a progressively type II censored sample distributed with the standard exponential distribution. Considering with the following transformations

$$
\left\{\begin{array}{l}
Z_{1}=n Y_{1} \\
Z_{2}=\left(n-r_{1}-1\right)\left(Y_{2}-Y_{1}\right) \\
Z_{3}=\left(n-r_{1}-r_{2}-2\right)\left(Y_{3}-Y_{2}\right) \\
\cdots \quad \cdots \\
Z_{m}=\left(n-r_{1}-\cdots-r_{m-1}-m+1\right)\left(Y_{m}-Y_{m-1}\right)
\end{array}\right.
$$

Viveros \& Balakrishnan [23] showed that the generalized spacings $Z_{1}, Z_{2}, \cdots, Z_{m}$ are all independent and identically distributed (i.i.d.) as standard exponential with mean 1 .

Then we have $2 \sum_{i=1}^{m} Z_{i} \sim \chi^{2}(2 m)$, that is

$$
\begin{aligned}
2 \sum_{i=1}^{m} Z_{i} & =2 \sum_{i=1}^{m}\left(1+r_{i}\right) Y_{i} \\
& =2 \sum_{i=1}^{m}\left(1+r_{i}\right) \theta \ln X_{i: m: n} \\
& =2 \theta T \sim \chi^{2}(2 m)
\end{aligned}
$$

Then it is easy to prove that the distribution of statistic $T$ is $\Gamma(m, \theta)$.

Theorem 1 The inverse linear estimator $(c T+d)^{-1}$ is 
admissible, provided $0 \leq c<c^{*}$ and $d>0$.

Proof. From the Bayes estimator in Eq. (12), we see that when $\alpha>0$ and $\beta>0$, the coefficient of $T$ is between 0 and $c^{*}$, and the constant $\frac{\beta}{n+\alpha-1}$ is strictly bigger than 0 . This proves that $(c T+d)^{-1}$ is admissible for the case $0<c<c^{*}$ and $d>0$. For the case $c=0, d>0$, the estimator $(c T+d)^{-1}$ is admissible since it is the unique estimator for which $R(\theta, d)=0$ when $\theta=d$.

Theorem 2 The inverse linear estimator $(c T+d)^{-1}$ is admissible, provided $c=c^{*}$ and $d \geq 0$.

Proof. We first consider the case $c=c^{*}$ and $d>0$. Let $\theta$ has the prior distribution $\pi_{k}(\theta)$ with pdf

$$
\pi_{k}(\theta)=\frac{\beta^{1 / \mathrm{k}}}{\Gamma(1 / \mathrm{k})} \theta^{1 / k-1} e^{-\beta \theta}, \beta>0, k>0
$$

If $A$ is a nondegenerate convex subset of $(0,+\infty)$, then it is obvious that there exists a $k_{0}$ such that $\int_{A} \pi_{k}(\lambda) d \lambda \geq \mathcal{E}$ for some $\varepsilon>0$ and all $k \geq k_{0}$.

In fact,

$$
\int_{A} \beta^{\frac{1}{k}} \theta^{\frac{1}{k}-1} e^{-\beta \theta} d \theta=\int_{A \cap(0,1)} \beta^{\frac{1}{k}} \theta^{\frac{1}{k}-1} e^{-\beta \theta} d \theta+\int_{A \cap[1,+\infty)} \beta^{\frac{1}{k}} \theta^{\frac{1}{k}-1} e^{-\beta \theta} d \theta
$$

When $k \rightarrow \infty$, we have $\beta^{\frac{1}{k}} \rightarrow 1$.

Then

$$
\begin{aligned}
\int_{A} \beta^{\frac{1}{k}} \theta^{\frac{1}{k}-1} e^{-\beta \theta} d \theta \geq & \frac{1}{2}\left[\int_{A \cap(0,1)} \theta^{\frac{1}{k}-1} \cdot e^{-\beta \theta} d \theta\right. \\
& \left.+\int_{A \cap[1,+\infty)} \theta^{\frac{1}{k}-1} \cdot e^{-\beta \theta} d \theta\right],\left(k>k_{0}\right)
\end{aligned}
$$

Let $\varepsilon_{0}=\frac{1}{2}\left[\int_{A \cap(0,1)} \theta^{\frac{1}{k}-1} \cdot e^{-\beta \theta} d \theta+\int_{A \cap[1,+\infty)} \theta^{\frac{1}{k}-1} \cdot e^{-\beta \theta} d \theta\right]$,

Then the conclusion has been proved.

The Bayes estimator with respect to prior distribution $\pi_{k}(\theta)$ under the entropy loss function (8) can be derived as in (12) as

$$
\hat{\theta}_{k}=\left(\frac{T+\beta}{n+1 / k-1}\right)^{-1}
$$

Let $\hat{\theta}(X)=\left(\frac{T+\beta}{n-1}\right)^{-1}, F(\theta, t)$ is the jiont probability density function of $(\theta, T)$.

Then $d F(\theta, t)=\frac{\beta^{\frac{1}{k}}}{\Gamma(1 / \mathrm{k})} \theta^{1 / k-1} e^{-\beta \theta} \cdot \frac{\theta^{n}}{\Gamma(n)} t^{n-1} e^{-\theta t} d \theta d t$

The difference of the Bayes risks with respect to $\hat{\theta}_{k}$ and $\hat{\theta}$ is

$$
\begin{aligned}
& r\left(\pi_{k}, \hat{\theta}\right)-r\left(\pi_{k}, \hat{\theta}_{k}\right)=E L(\hat{\theta}, \theta)-E L\left(\hat{\theta}_{k}, \theta\right) \\
& =\int_{0}^{+\infty} \int_{0}^{+\infty}\left(\frac{\hat{\theta}}{\theta}-\ln \frac{\hat{\theta}}{\theta}-1\right)-\left(\frac{\hat{\theta}_{k}}{\theta}-\ln \frac{\hat{\theta}_{k}}{\theta}-1\right) d F(\theta, t) \\
& =p(k) \int_{0}^{+\infty} \int_{0}^{+\infty} \frac{1}{\theta(T+\beta)} d F(\theta, t)-q(k)
\end{aligned}
$$

Here $\quad p(k)=\frac{-1}{k}, q(k)=\ln \frac{n-1}{n+1 / k-1} \quad$ and $\quad p(k) \rightarrow 0$ $q(k) \rightarrow 0, k \rightarrow+\infty$

For any prior Gamma distribution $\Gamma(\alpha, \beta)$ of $\theta$, we have

$$
\begin{aligned}
& E\left[\frac{1}{\theta(T+\beta)}\right] \leq E\left[\frac{1}{\theta T}\right] \\
& =\int_{0}^{+\infty} \int_{0}^{+\infty} \frac{1}{\theta t} \cdot \frac{t^{n-1}}{\Gamma(n)} \theta^{n} e^{-\theta t} \cdot \frac{\beta^{\alpha}}{\Gamma(\alpha)} \theta^{\alpha-1} e^{-\beta \theta} d \theta d t \\
& =\int_{0}^{+\infty}\left[\int_{0}^{+\infty} t^{(n-1)-1} e^{-\theta t} d t\right] \cdot \frac{\beta^{\alpha}}{\Gamma(\alpha) \Gamma(n)} \theta^{(n+\alpha-1)-1} e^{-\beta \theta} d \theta \\
& =\int_{0}^{+\infty} \frac{\Gamma(n-1)}{\theta^{n-1}} \cdot \frac{\beta^{\alpha}}{\Gamma(\alpha) \Gamma(n)} \theta^{(n+\alpha-1)-1} e^{-\beta \theta} d \theta \\
& =\frac{\Gamma(n-1) \cdot \beta^{\alpha}}{\Gamma(\alpha) \Gamma(n)} \cdot \frac{\Gamma(\alpha)}{\beta^{\alpha}}=\frac{1}{n-1}<+\infty
\end{aligned}
$$

Thus $\lim _{k \rightarrow \infty}\left(r\left(\pi_{k}, \hat{\theta}\right)-r\left(\pi_{k}, \hat{\theta}_{k}\right)\right)=0$,

Therefore, $(c T+d)^{-1}$ is admissible for any $\beta>0$ by Blyth's lemma. This proves that the inverse linear estimator $(c T+d)^{-1}$ is admissible for $c=c^{*}$ and $d>0$.

For the case $c=c^{*}$ and $d=0,\left(c^{*} T\right)^{-1}$ is the limit of Bayes estimator relative to the gamma prior $\Gamma(\alpha, \beta)$, as $\alpha, \beta \rightarrow 0$. It is easy to verify that the Bayes risk difference $r\left(\pi_{k},\left(c^{*} T\right)^{-1}\right)-r\left(\pi_{k}, \hat{\theta}_{B}\right)$ converges to zero as $\alpha, \beta \rightarrow 0$. Then we prove the admissibility of $\left(c^{*} T\right)^{-1}$.

Theorem 3 Let the parameter space be $(0,+\infty)$ and the action space be $[0,+\infty)$. The inverse linear estimator $(c T+d)^{-1}$ is inadmissible under the entropy loss function (8) whenever one of the following conditions holds:

(i) $c<0$ or $d<0$;

(ii) $0<c \neq c^{*}, d=0$

(iii) $c>c^{*}$ and $d \geq 0$

Proof. For the case (i), because $(c T+d)^{-1}$ takes on negative values with positive probability. Therefore $(c T+d)^{-1}$ is dominated by the estimator $\max \left(0,(c T+d)^{-1}\right)$. Then (i) is proved.

For the case (ii), the risk function of the estimator $(c T)^{-1}$ is

$$
\begin{aligned}
R\left(\theta,(c T)^{-1}\right) & =E\left[\frac{1}{\theta(c T)}-\ln \frac{1}{\theta(c T)}-1\right] \\
& =\frac{1}{c \theta} E \frac{1}{T}+E(\ln T)+\ln \theta+\ln c-1
\end{aligned}
$$


Using Lemma 2, we have

$$
E \frac{1}{T}=\frac{\theta}{n-1}, E(\ln T)=\psi(n)-\ln \theta,
$$

where $\psi(n)=\frac{\Gamma^{\prime}(n)}{\Gamma(n)}$.

The derivative of the risk function with respect to $c$ is

$$
\frac{\partial}{\partial c} R\left(\theta,(c T)^{-1}\right)=-\frac{1}{c^{2}} \frac{1}{n-1}+\frac{1}{c}=\frac{1}{c}\left(1-\frac{1 / c}{n-1}\right)
$$

Then $\frac{\partial}{\partial c} R\left(\theta,(c T)^{-1}\right)<0$ where $0<c<c^{*}=\frac{1}{n-1}$, and $\frac{\partial}{\partial c} R\left(\theta,(c T)^{-1}\right)>0$, where $c>c^{*}$.

Thus the risk function of $(c T)^{-1}$ is minimized at the value $c=c^{*}$. Hence the estimator $(c T)^{-1}$ is dominated by the estimator $\left(c^{*} T\right)^{-1}$. Thus (ii) is proved.

For the case (iii). Because

$$
\begin{aligned}
& R\left(\theta,(c T+d)^{-1}\right)-R\left(\theta^{*},\left(c^{*} T+\frac{c^{*}}{c} d\right)^{-1}\right) \\
& =E\left[\frac{1}{\theta(c T+d)}-\ln \frac{1}{\theta(c T+d)}-\frac{1}{\theta\left(c^{*} T+\frac{c^{*}}{c} d\right)}+\frac{1}{\theta\left(c^{*} T+\frac{c^{*}}{c} d\right)}\right]
\end{aligned}
$$

Under the condition (iii), $1-\frac{c}{c^{*}}<0$, then

$$
\begin{gathered}
R\left(\theta,(c T+d)^{-1}\right)-R\left(\theta^{*},\left(c^{*} T+\frac{c^{*}}{c} d\right)^{-1}\right) \\
=\left\{E\left[\frac{1}{\theta(c T+d)}\left(1-\frac{c}{c^{*}}\right)+\ln \frac{c}{c^{*}}\right]\right\}, \\
\left.\geq\left\{\left(1-\frac{c}{c^{*}}\right) E\left[\frac{1}{\theta c T}\right]+\ln \frac{c}{c^{*}}\right]\right\}=n\left(\frac{c^{*}}{c}-\ln \frac{c^{*}}{c}-1\right)>0
\end{gathered}
$$

Therefore, $R\left(\theta,(c T+d)^{-1}\right)$ is minimized at $c=c^{*}$. Hence $(c T+d)^{-1}$ is dominated by $\left(c^{*} T+d\right)^{-1}$ in this case.

Remark 3. The MLE $\hat{\theta}_{M L E}$ and Bayes estimator $\hat{\theta}_{E B}$ are inadmissible by using Theorem 3. They are both dominated by the generalized Bayes estimator $\hat{\theta}_{*}=\left(c^{*} T\right)^{-1}$.

\section{Conclusions}

This paper considers the estimation of the unknown parameter of Pareto distribution based on progressively type II censored samples. The MLE, Bayes estimator and empirical Bayes estimators are obtained. These estimators all belong to a class of inverse linear estimators with the form $(c T+d)^{-1}$, where $T=\sum_{i=1}^{m}\left(1+r_{i}\right) \ln X_{i: m: n}$. The admissibility and inadmissibility of $(c T+d)^{-1}$ are discussed. As a result, the MLE and empirical Bayes estimator are inadmissible.

\section{Acknowledgement}

This study is partially supported by Natural Science Foundation of Hunan Province (No. 2016JJ4012 and No. 2015JJ3030) and Foundation of Hunan Educational Committee (No.15C0228).

\section{References}

[1] Cramer E., Navarro J., 2015. Progressive Type-II censoring and coherent systems. Naval Research Logistics, 62(6):512530 .

[2] Bakoban, R. A., 2015. Parameters estimation of the generalized inverted Rayleigh distribution based on progressive type II censoring. Advances \& Applications in Statistics, 47(1): 19-50.

[3] Ng H. K. T., Chan P. S., Balakrishnan N., 2004. Optimal progressive censoring plans for the Weibull distribution. Technometrics, 46(46):470-481.

[4] Soliman A. A., Abd-Ellah A. H., Abou-Elheggag N. A., Ahmed E. A., 2012. Modified weibull model: a Bayes study using MCMC approach based on progressive censoring data. Reliability Engineering and System Safety, 100(2): 48-57.

[5] Yang C., 2000. Statistical analysis of Weibull distributed lifetime data under Type II progressive censoring with binomial removals. Journal of Applied Statistics, 27(8):10331043.

[6] Wu S. J., 2008. Estimation of the two-parameter bathtubshaped lifetime distribution with progressive censoring[J]. Journal of Applied Statistics, 35(10):1139-1150.

[7] Cho Y., Sun H., Lee K., 2015. Estimating the entropy of a Weibull distribution under generalized progressive hybrid censoring. Entropy, 17(1):102-122.

[8] Bhattacharya R., Pradhan B., Dewanji A., 2016. On optimum life-testing plans under Type-II progressive censoring scheme using variable neighborhood search algorithm. Test, 25(2):1-22.

[9] Laumen B., Cramer E., 2015. Likelihood inference for the lifetime performance index under progressive type-II censoring. Economic Quality Control, 30(2):59-73.

[10] Khorram E., Farahani Z. S. M., 2016. Statistical inference of weighted exponential lifetimes under progressive type-II censoring scheme. Quality Technology \& Quantitative Management, 11(4):433-451.

[11] Wu S. J., Chang C. T., 2003. Inference in the Pareto distribution based on progressive Type II censoring with random removals. Journal of Applied Statistics, 30(2):163-172.

[12] Raqab M. Z., Asgharzadeh A., Valiollahi R., 2010. Prediction for Pareto distribution based on progressively Type-II censored samples. Computational Statistics \& Data Analysis, 54(54):1732-1743.

[13] Kulldorff G., Vannman K., 2012. Estimation of the location and scale parameters of a Pareto distribution by linear functions of order statistics. Journal of the American Statistical Association, 68(68):218-227. 
[14] Fu J., Xu A., Tang Y., 2012. Objective Bayesian analysis of Pareto distribution under progressive Type-II censoring. Statistics \& Probability Letters, 82(10): 1829-1836.

[15] Saldaña-Zepeda D. P., Vaquera-Huerta H., Arnold B. C., 2010. A goodness of fit test for the Pareto distribution in the presence of Type II censoring, based on the cumulative hazard function. Computational Statistics \& Data Analysis, 54(4): 833-842.

[16] Wen D. L., Levy M. S., 2006. Admissibility of Bayes estimates under BLINEX loss for the normal mean problem. Communications in Statistics-Theory and Methods, 30(1): 155-163.

[17] Zakerzadeh H., Zahraie S. H. M., 2015. Admissibility in nonregular family under squared-log error loss. Metrika, 78(2): 227-236.

[18] Hara, H., Takemura, A., 2009. Bayes admissible estimation of the means in poisson decomposable graphical models. Journal of Statistical Planning \& Inference, 139(4): 1297-1319.
[19] Mahmoudi E., 2012. Admissible and minimax estimators of $\theta$ with truncated parameter space under squared-log error loss function. Communication in Statistics-Theory and Methods, 41(7): 1242-1253.

[20] Nancy R. M., 1971. Best linear invariant estimation for Weibull parameters under progressive censoring. Technometrics, 13(3): 521-533.

[21] Balakrishnan, N. and Aggarwala, R., 2000. Progressive Censoring: Theory, Method and Applications. Boston: Birkhauser Publishers.

[22] Wang D., Song L., Wang D, et al., 1999. Estimation of the exponential distribution parameter under an entropy loss function based on type II censoring. Chinese Journal of Applied Probability \& Statisties, 15(15): 176-186.

[23] Viveros R., Balakrishnan N., 1994. Interval estimation of parameters of life from progressively censored data. Technometrics, 36(1): 84-91. 